\title{
Phase 1/2a clinical trial of gene-corrected autologous cell therapy for recessive dystrophic epidermolysis bullosa
}

Shaundra Eichstadt, ${ }^{1}$ Melissa Barriga, ${ }^{1}$ Anusha Ponakala, ${ }^{1}$ Claudia Teng, ${ }^{1}$ Ngon T. Nguyen, ${ }^{2}$ Zurab Siprashvili, ${ }^{1}$ Jaron Nazaroff,, ${ }^{1}$ Emily S. Gorell, ${ }^{1}$ Albert S. Chiou, ${ }^{1}$ Lisa Taylor, ${ }^{3}$ Phuong Khuu, ${ }^{3}$ Douglas R. Keene, ${ }^{4}$ Kerri Rieger, ${ }^{1}$ Rohit K. Khosla, ${ }^{1}$ Louise K. Furukawa, ${ }^{3}$ H. Peter Lorenz, ${ }^{3}$ M. Peter Marinkovich, ${ }^{1,5}$ and Jean Y. Tang ${ }^{1}$

'Stanford University School of Medicine, Department of Dermatology, Redwood City, California, USA. ${ }^{2}$ Abeona

Therapeutics, Cleveland, Ohio, USA. 'ucile Packard Children's Hospital, Stanford Children's Health, Stanford, California, USA. ${ }^{4}$ Shriners Hospital for Children, Portland, Oregon, USA. ${ }^{5}$ Veterans Affairs Medical Center, Palo Alto, California, USA.

BACKGROUND. Recessive dystrophic epidermolysis bullosa (RDEB) patients have mutations in the COL7A1 gene and thus lack functional type VII collagen (C7) protein; they have marked skin fragility and blistering. This single-center phase $1 / 2$ a open-label study evaluated the long-term efficacy, safety, and patient-reported outcomes in RDEB patients treated with gene-corrected autologous cell therapy.

METHODS. Autologous keratinocytes were isolated from participant skin biopsies. Epidermal sheets were prepared from cells transduced with a retrovirus carrying the full-length human COL7A1 gene. These gene-corrected autologous epidermal sheets measured $5 \times 7 \mathrm{~cm}\left(35 \mathrm{~cm}^{2}\right)$ and were transplanted onto 6 wound sites in each of 7 adult participants ( $n=42$ sites total) from 2013 to 2017 . Participants were followed for 2 to 5 years.

RESULTS. No participants experienced any serious related adverse events. Wound healing of $50 \%$ or greater by Investigator Clobal Assessment was present in $95 \%$ (36 of 38) of treated wounds versus $0 \%(0$ of 6$)$ of untreated control wounds at 6 months $(P<0.0001)$. At year $1,68 \%$ (26 of 38) of treated wounds had $50 \%$ or greater healing compared with $17 \%$ (1 of 6$)$ of control wounds $(P=$ 0.025). At year $2,71 \%$ ( 27 of 38 ) of treated wounds had $50 \%$ or greater healing compared with $17 \%$ (1 of 6) of control wounds $(P=0.019)$.

CONCLUSION. C7 expression persisted up to 2 years after treatment in 2 participants. Treated wounds with $50 \%$ or greater healing demonstrated improvement in patient-reported pain, itch, and wound durability. This study provides additional data to support the clinically meaningful benefit of treating chronic RDEB wounds with ex vivo, $\mathrm{C7}$ gene-corrected autologous cell therapy. This approach was safe and promoted wound healing that was associated with improved patientreported outcomes.

TRIAL RECISTRATION. Clinicaltrials.gov identifier: NCT01263379.

FUNDING. Epidermolysis Bullosa Research Partnership, Epidermolysis Bullosa Medical Research Foundation, NIH R01 AR055914, Office of Research and Development at the Palo Alto Veteran's Affairs Medical Center, and the Dermatology Foundation.

declared that no conflict of interest exists.

Copyright: ( 2019 , American Society for Clinical Investigation.

Submitted: June 7, 2019

Accepted: August 31, 2019

Published: October 3, 2019 .

Reference information: JCI Insight. 2019;4(19):e130554

https://doi.org/10.1172/jici.

insight.130554.

\section{Introduction}

Recessive dystrophic epidermolysis bullosa (RDEB) is a rare, life-threatening bullous genodermatosis $(1,2)$. Genetic mutations in the COL7A1 gene lead to lack of functional type VII collagen (C7), a large triple-helical protein found beneath the lamina densa (2-4). C7 contains 2 noncollagenous domains (NC1 and NC2) and a central collagenous domain, forming anchoring fibrils (AFs) that are critical to dermal-epidermal basement cohesion $(2,4)$. Mutations in COL7A1 lead to disruptions in keratinocyte adhesion, reducing mucocu- 
Table 1. Baseline characteristics of seven adult RDEB participants

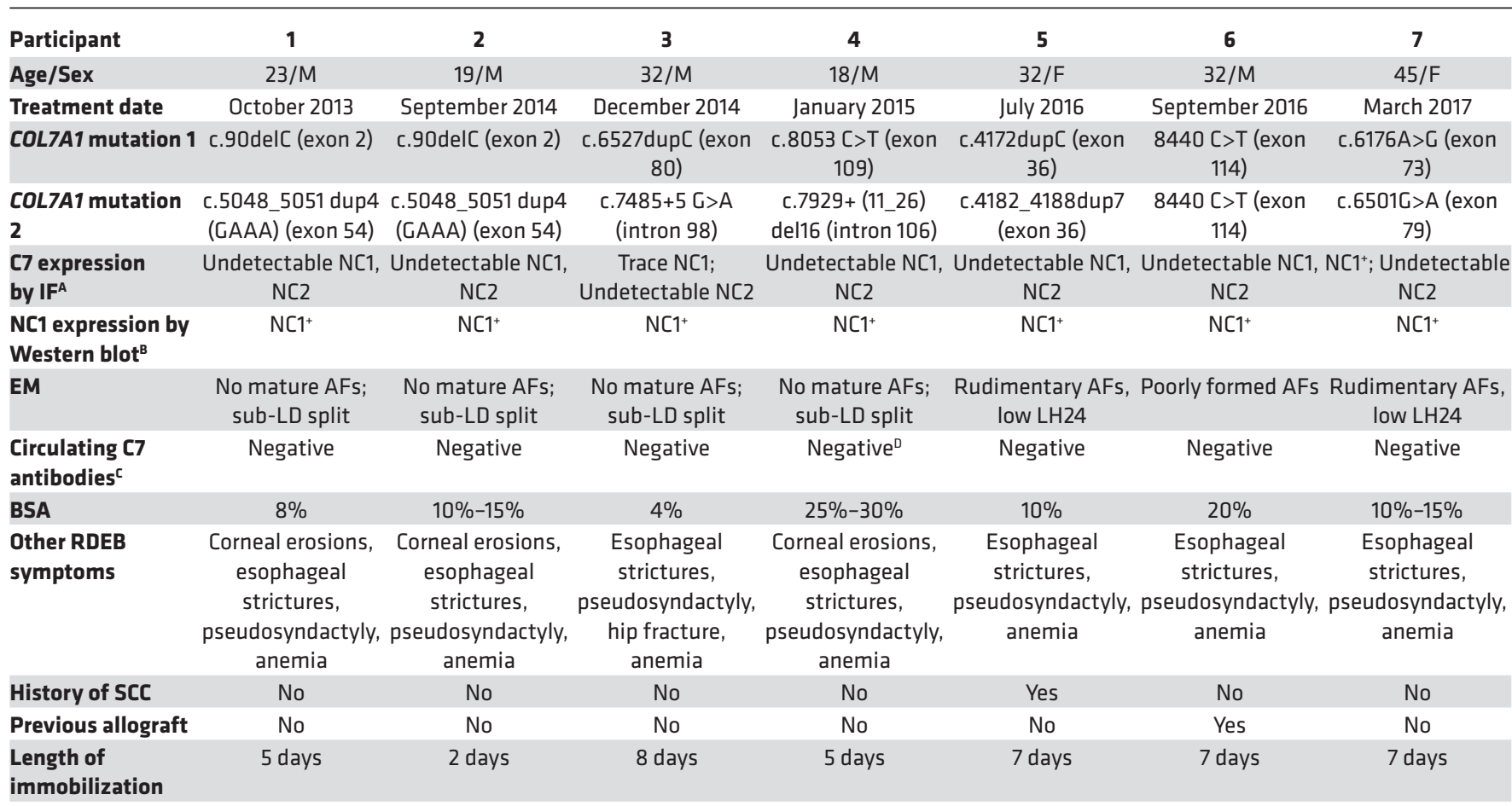

${ }^{A}$ Assessed using the LH7.2 antibody to detect NC1 and the LH24 antibody to detect NC2. ${ }^{B}$ Skin biopsy sample evaluated using the FNC1 antibody. ${ }^{\text {CAssayed }}$ by indirect immunofluorescence using patient serum placed on monkey esophagus to detect $C 7$ antibodies localized to dermal-epidermal junction. ${ }^{\circ}$ Circulating $\mathrm{C7}$ antibodies seen on Western blot at baseline for participant 4, examined following evidence of immune response after treatment. BSA, estimated wounded body surface area; SCC, squamous cell carcinoma; IF, immunofluorescence of skin biopsy; EM, electron microscopy of skin biopsy; AF anchoring fibrils; LD, lamina densa; C7, type VII collagen; NC, noncollagenous domain.

taneous mechanical stability $(3,5,6)$. RDEB is thus characterized by skin fragility, blistering, chronic and recurrent wounds with significant pain and pruritus, frequent wound infections, as well as restrictive scarring, resulting in pseudosyndactyly (2, 7-9). Patients suffer from recurrent wounds, which heal and re-blister, as well as chronic open wounds that are present for at least 12 weeks, often for many years (10).

Current treatment is strictly palliative, consisting of extensive wound care, treatment of infections, and prevention of skin trauma. Prior studies of allografts produced only short-term healing of several weeks in RDEB wounds (10-13). No effective treatments exist as disease-modifying therapy for RDEB wounds, leaving patients predisposed to developing aggressive squamous cell carcinomas (SCCs), the most common cause of death in RDEB patients (8).

We developed autologous keratinocyte sheets expressing full-length $\mathrm{C} 7$ using a retroviral vector (LZRSE) containing the COL7A1 gene (14). We previously reported methods and initial results for the first 4 RDEB participants treated with this therapy (6) and now present long-term follow-up results for a total of 7 RDEB participants with 42 treated wounds.

\section{Results}

Participants and treatment. Seven participants with severe generalized RDEB were enrolled, including the 4 previously reported (6), with a mean age of 28.7 years at time of treatment and with an estimated wounded body surface area range of 4\%-30\% (Table 1). Wounds selected for treatment had been present for a mean of 11.2 years (range: $3-20$ years).

Findings following intervention. All 42 treated sites were serially monitored for wound healing, infection, pain, itch, durability, and ease of blistering. Figure 1 shows representative clinical photographs of wound healing from participant 5 (left arm) and participant 7 (back) at baseline and at months 3, 6, 12, and 24 as well as a control chronic wound from participant 5 (right arm). 
A

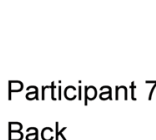

Back
Baseline

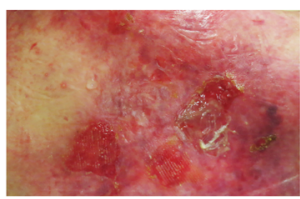

Month 3

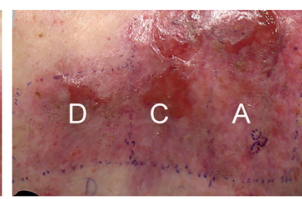

Month 6

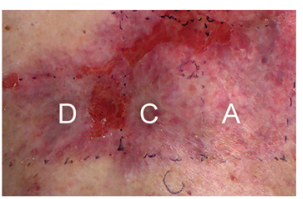

Month 12

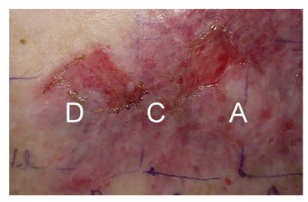

Month 24

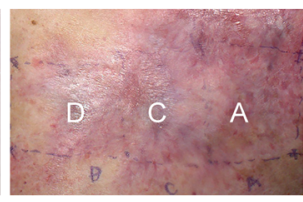

\section{B}

Participant 5

Right Arm

Control Wound

\section{Baseline}

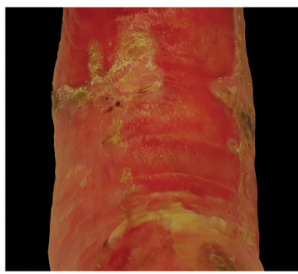

Month 3

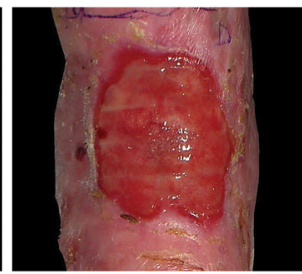

Month 6

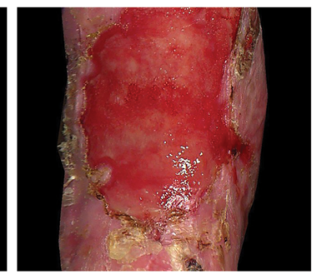

Month 12

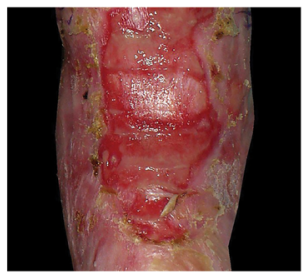

Month 24

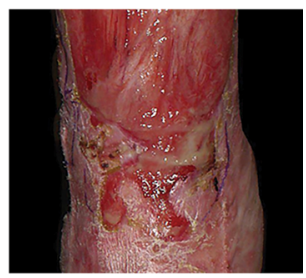

\section{C}

Participant 5 Left Arm

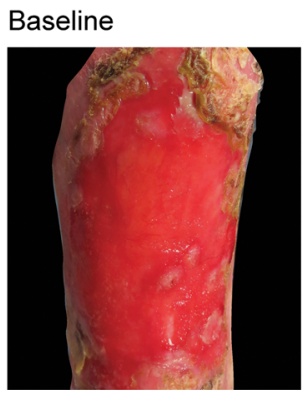

\section{Month 3}

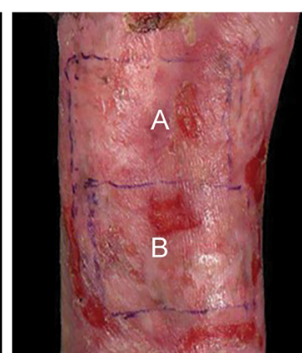

Month 6

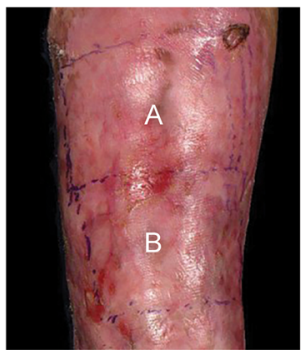

Month 12

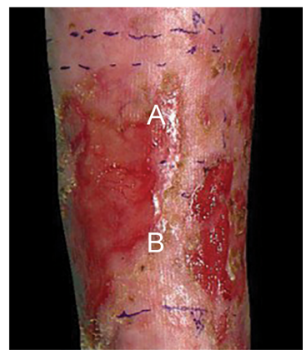

Month 24

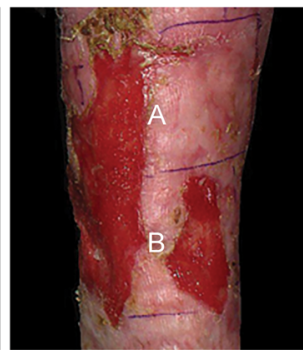

Figure 1. Representative clinical photographs of $C 7$ gene-corrected cell therapy in participants. (A) Participant 7, C7 gene-corrected treated sites $A, C$, and $D$, upper back. (B) Participant 5, untreated control wound, right antecubital fossa. (C) Participant 5, C7 gene-corrected treated sites A and B, left upper arm. Photographs of RDEB wounds at baseline and at designated time points after treatment. In $\mathbf{A}$ and $\mathbf{C}$, purple marker outlines gene-corrected autologous cellular sheet edges; each treated wound is denoted with a letter. Participant 7's sites A, C, and D demonstrate persistent healing at 2 years after treatment (A). Participant 5's sites A and B (C) demonstrated wound healing at months 3 and 6. Participant 5's untreated control wound (B) did not close during the measured time points.

Figure 2 is a summary table of Investigator Global Assessment (IGA) of wound healing of each treated site at each follow-up visit. As seen in Figures 2 and 3, at 3 and 6 months, 95\% (36 of 38) of treated sites had $50 \%$ or greater healing, compared with $68 \%$ (26 of 38 ) at 12 months, $71 \%$ (27 of 38) at 2 years, and $80 \%$ (16 of 20) at 3 years. Similarly, treated sites demonstrated $75 \%$ or greater wound healing most reliably during the early months following treatment: $82 \%$ (31 of 38) at 3 months, $66 \%$ ( 25 of 38) at 6 months, 39\% (15 of 38) at 12 months, $58 \%$ (22 of 38) at 2 years, and $70 \%$ (14 of 20) at 3 years.

Wound healing of $50 \%$ or greater by IGA was present in $95 \%$ (36 of 38) of treated wounds versus $0 \%$ ( 0 of 6 ) of untreated control wounds at 6 months $(P<0.0001)$. At year $1,68 \%$ (26 of 38) of treated wounds had $50 \%$ or greater healing compared with $17 \%$ (1 of 6$)$ of control wounds $(P=0.025)$. At year $2,71 \%(27$ of 38$)$ of treated wounds had $50 \%$ or greater healing compared with $17 \%$ ( 1 of 6$)$ of control wounds $(P=0.019)$ (Figure 3 ).

At 12 months, 3 out of 7 participants were positive for either NC2 or AFs, indicating restoration of C7 (Table 2). At 2 years, 2 out of 3 participants with biopsies were positive for either NC2 or AFs (one participant was positive for both $\mathrm{AF}$ and $\mathrm{C} 7$; the second participant was positive only for $\mathrm{C} 7$, as an additional biopsy for $\mathrm{AF}$ assessment was not obtained).

The NC2 domain of C7 was detected in 73\% (11 of 15) of biopsies at 3 months, in 53\% (8 of 15) of biopsies at 6 months, in 11\% ( 1 of 9) of biopsies at 1 year, and 67\% ( 2 of 3 ) at 2 years. AFs were seen in $67 \%$ ( 8 of 12) of biopsies at 3 months, in 53\% (8 of 15) of biopsies at 6 months, in $43 \%$ (3 of 7) of biopsies at 1 year, and $50 \%$ ( 1 of 2 ) of biopsies at 2 years. Figure 4 shows representative immunofluorescence (IF) and immuno-electron microscopy (IEM) images from skin biopsies of treated sites on participants 4 and 6. C7 was localized by IF and by electron microscopy (EM) to ultrastructurally recognizable AFs at the epithelial basement membrane at year 1 in participant 6 and year 2 in participant 4 .

Prior to treatment, participants reported pain in 53\% (20 of 38) of wound sites. At treated sites with $50 \%$ or greater wound healing, participants reported pain in $0 \%$ ( 0 of 26$)$ of sites at 1 year, $4 \%$ ( 1 of 27 ) at 2 years, 


\begin{tabular}{|c|c|c|c|c|c|c|c|c|c|c|}
\hline Participant & Site & Location & $\begin{array}{l}\text { Wound Age } \\
\text { (years) }\end{array}$ & 3 mos. & 6 mos. & 12 mos. & 2 years & 3 years & 4 years & 5 years \\
\hline \multirow{5}{*}{1} & A & $\mathrm{R}$ distal forearm & $>5$ & & & & & & & \\
\hline & $\mathrm{B}$ & L forearm & $>5$ & & & & & & & \\
\hline & $\mathrm{D}$ & R shoulder & $>5$ & & & & & & & \\
\hline & $\mathrm{E}$ & $\mathrm{L}$ arm & $<1$ & & & & & & & \\
\hline & $Z$ & $\mathrm{R}$ arm & Induced & & & & & & & \\
\hline \multirow[t]{4}{*}{2} & $\mathrm{C}$ & $\mathrm{R}$ forearm & $3-5$ & & & & & & & \\
\hline & $\mathrm{D}$ & R posterior shoulder & $>5$ & & & & & & & \\
\hline & $\mathrm{E}$ & Lower back & $>5$ & & & & & & & \\
\hline & $\bar{Z}$ & R upper chest & Induced & & & & & & & \\
\hline \multirow{2}{*}{3} & $\mathrm{~A}$ & R lateral hand & $3-5$ & & & & & & & \\
\hline & $\mathrm{B}$ & R medial hand & $3-5$ & & & & & & & \\
\hline \multirow{5}{*}{4} & $\mathrm{~B}$ & $\mathrm{~L}$ medial forearm & $>5$ & & & & & & & \\
\hline & $\mathrm{C}$ & L proximal forearm & $>5$ & & & & & & & \\
\hline & $\mathrm{D}$ & $\mathrm{R}$ lateral forearm & $>5$ & & & & & & & \\
\hline & $E$ & $\mathrm{R}$ distal forearm & $>5$ & & & & & & & \\
\hline & $Z$ & R medial forearm & Induced & & & & & & & \\
\hline \multirow{6}{*}{5} & $\mathrm{~A}$ & L upper arm & 16 & & & & & & & \\
\hline & $\mathrm{B}$ & L upper arm & 16 & & & & & & & \\
\hline & $\mathrm{C}$ & $\mathrm{R}$ upper arm & 16 & & & & & & & \\
\hline & $\mathrm{D}$ & $\mathrm{R}$ upper arm & 16 & & & & & & & \\
\hline & $\mathrm{E}$ & $\mathrm{R}$ upper arm & 16 & & & & & & & \\
\hline & $\mathrm{F}$ & R upper arm & 16 & & & & & & & \\
\hline 6 & $\mathrm{~A}$ & R lateral back upper & 20 & & & & & & & \\
\hline \multirow{2}{*}{7} & $\mathrm{E}$ & $\mathrm{R}$ foot front anterior & 20 & & & & & & & \\
\hline & $\mathrm{F}$ & Back upper corner & 20 & & & & & & & \\
\hline
\end{tabular}

R: right, L: left, blank spaces denote prospective dates

Figure 2. Clinical response of wound healing as assessed by investigator global assessment (IGA). Percentage of wound healing based on clinical assessment by IGA at designated time points. A green box indicates $\geq 75 \%$ wound healing, yellow box indicates $50 \%-74 \%$ wound healing, and a red box indicates $\leq 49 \%$ wound healing. R, right; L, left. Blank white spaces denote prospective dates.

and $0 \%(0$ of 16$)$ at 3 years. Similarly, participants reported improvement of itch compared with baseline. Prior to treatment, itch was present in $61 \%$ (23 of 38) of wounds compared with 19\% (5 of 26) of treated sites at 1 year, $7 \%$ ( 2 of 27 ) at 2 years, and $0 \%(0$ of 16$)$ at 3 years. Participants also reported improved durability of treated wounds compared with the wounds at baseline in $82 \%$ (31 of 38) at 1 year, $81 \%$ (30 of 37) at 2 years, and $70 \%$ (14 of 20) at 3 years. Similarly, blistering was reported as more difficult in $82 \%$ (31 of 38) of treated wounds at 1 year, $81 \%$ (30 of 37) at 2 years, and $75 \%$ (15 of 20) at 3 years. These outcome measures were based on participant self-report at each of the treated sites. All 7 participants reported they would undergo the treatment again and would recommend the procedure to other patients with RDEB.

Safety. No serious related adverse events were reported. There was no evidence of systemic immune symptoms and no development of SCC at any treated site. Replication-competent retrovirus (RCR) and cytotoxic $\mathrm{T}$ cell assays were negative at all time points assayed (Supplemental Table 1; supplemental material available online with this article; https://doi.org/10.1172/jci.insight.130554DS1). The most common related adverse events were infection of treated site $(n=2)$, pruritus around the treated site $(n=3)$, and pain 
A

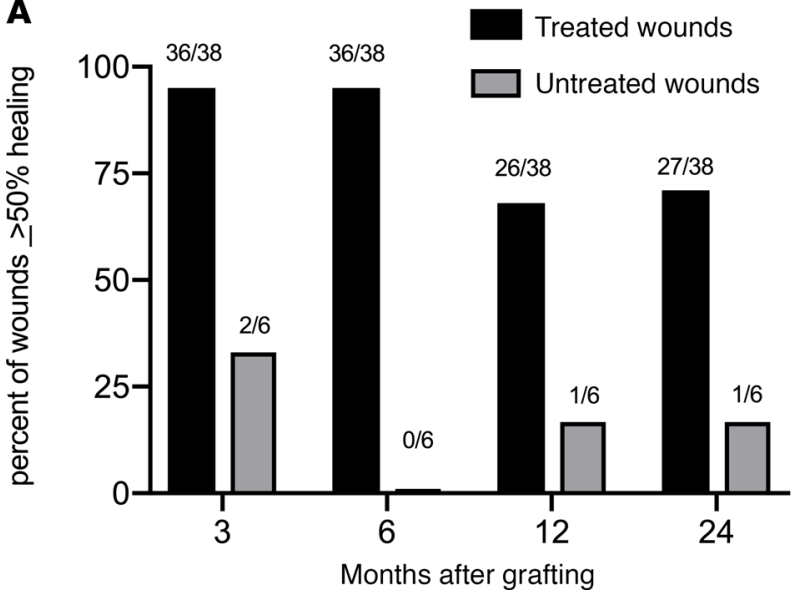

B

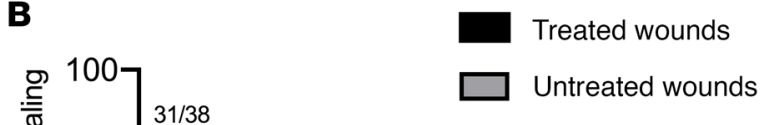

Figure 3. Wound healing in treated and untreated wounds. Summary of treated wounds and untreated control wounds that achieved $\geq 50 \%$ wound healing (A) and wounds that achieved $\geq 75 \%$ wound healing (B) at designated time points after treatment, as assessed by Investigator Clobal Assessment. Black bars represent treated sites; light gray bars represent untreated control wounds.

around the treated site $(n=1)$ (Supplemental Table 2). Local wound infections were treated with topical or oral antibiotics; no participants developed systemic infections.

One of participant 4's treated sites demonstrated tissue-bound $\operatorname{IgG}$ antibodies on direct immunofluorescence (DIF) at 3 months (6) and 2 years, suggesting a persistent localized immune response with anti-C7 antibodies. However, as we previously reported (6), this participant had transient anti-C7 circulating antibodies at months 1,3 , and 6 . Circulating antibodies were not detectable thereafter, suggesting this participant's immune response was localized rather than systemic. Additional analysis of pretreatment serum samples demonstrated low levels of anti-C7 antibodies (1:300) detected only by a more sensitive Western blot assay, and were directed against epitopes at or near the NC2 domain (ref. 6 and Supplemental Table 1). No other signs or symptoms suggestive of a systemic immune response, such as new-onset generalized blistering, occurred.

Two participants developed SCCs during the study period, one of whom had a prior history of SCC (participant 5). All tumors were located at sites distant to treated sites. Participant 5 developed 1 SCC during the study period, which was resected and found to be negative for retrovirus by PCR. Participant 6 developed 3 SCCs during the study period, including 1 metastatic to regional lymph nodes. One of participant 6's tumors was available for analysis and was negative for retrovirus. The participants were 32 and 34 years old, respectively, at the time of their first SCC diagnosis during the study period.

\section{Discussion}

The long-term follow-up data from this phase $1 / 2 \mathrm{a}$ trial of 7 RDEB participants suggest that treatment of chronic open wounds with C7 gene-corrected autologous cell therapy is safe and may represent the first corrective therapy for long-standing RDEB wounds. Greater-than-50\% wound healing was associated with improvement in patient-reported outcomes. Significant wound healing of greater than $50 \%$ occurred most commonly during the first 6 months following treatment, when $95 \%$ of treated sites remained at least $50 \%$ healed. Importantly, the majority of treated sites continued to remain at least $50 \%$ healed at 2 years $(71 \%)$ and 3 years $(80 \%)$ after treatment. Treated wounds had significantly improved rates of healing compared with untreated control wounds at 6 months, 1 year, and 2 years. Participants also reported sustained improvement at treated sites in pain, itch, durability, and a reduced ease of blistering at treated sites at all measured time points.

Evaluation of $\mathrm{C} 7$ molecular correction by biopsy can be challenging, and not all biopsies of treated sites were positive for the NC2 domain of $\mathrm{C} 7$ and/or AFs. Biopsy results could be affected by site-selection bias or driven by heterogeneous expression of $\mathrm{C} 7$ due to infiltration of noncorrected native epidermal stem cells that persist despite surgical preparation of the wound bed before treatment (15). Also, while many previous RDEB studies examined only the $\mathrm{C} 7 \mathrm{NC} 1$ domain, we focused on the $\mathrm{NC} 2$ domain, which is more indicative of 
Table 2. Molecular correction of type VII collagen by participant and study follow-up

\begin{tabular}{|c|c|c|c|c|c|c|c|c|}
\hline \multirow[t]{2}{*}{ Participant } & \multicolumn{2}{|c|}{3 months } & \multicolumn{2}{|c|}{6 months } & \multicolumn{2}{|c|}{12 months } & \multicolumn{2}{|c|}{24 months } \\
\hline & $\mathrm{NC2}$ & $\mathrm{AF}$ & NC2 & $A F$ & NC2 & AF & NC2 & AF \\
\hline 1 & + & - & + & + & + & + & - & $-A$ \\
\hline 2 & + & + & - & + & - & - & ND & ND \\
\hline 3 & + & + & + & + & - & $-{ }^{A}$ & ND & ND \\
\hline 4 & + & + & $-A$ & - & - & + & + & + \\
\hline 5 & + & + & + & - & - & - & ND & ND \\
\hline 6 & + & + & + & + & $+^{B}$ & + & + & ND \\
\hline 7 & + & + & + & + & $N D^{C}$ & $N D^{C}$ & ND & ND \\
\hline
\end{tabular}

For each participant, molecular correction of type VII collagen (C7) was assessed by both immunofluorescence and presence of anchoring fibrils. The NC2 domain of $\mathrm{C} 7$ was assessed using the LH24 antibody. Presence of anchoring fibrils (AFs) was assessed by immunoelectron microscopy. This table depicts whether NC2 or AFs were present in any skin biopsy sample at a given time point. Samples were obtained at 3 months, 6 months, 12 months, and 24 months after treatment. ${ }^{A} F N C 1$ positive. ${ }^{B}$ Biopsy was obtained at 9 months. ${ }^{C}$ No epidermis was present in the sample. ND, not done; AF, anchoring fibrils present; NC2, noncollagenous domain of type VII collagen present.

full-length C7 expression (6). Despite these limitations, continued expression of the NC2 domain was detected in biopsies of treated sites as late as 2 years after treatment in participants 4 and 6 . Additionally, AFs were seen on EM in participants 1, 4, and 6 on year 1 biopsies and on year 2 biopsy for participant 4 . Thus, durability of C7 molecular expression appeared to be an important factor in achieving durability of wound healing.

C7 expression levels of our participants' treated sites were not quantified nor directly compared to normal human skin. However, we estimate that at 3 months, the level of $\mathrm{C} 7$ expression is $50 \%$ compared with normal skin based on the images presented in Figure 4 (14).

A recent paper on intradermal injections of autologous lentivirus-corrected fibroblasts in RDEB patients also describes increased expression of C7 (16); however, this was not translated into mature AF formation. One participant described subjective improvement in biopsy-site healing in that study; however, corrected fibroblasts were injected into intact skin and not chronically wounded areas. Thus, it is unclear how these injections would translate into effects on wound healing. Additional data on improvements in durability and reduced tendency to blister were not reported, as we have seen in our study. Further investigation is necessary to determine the molecular factors associated with improved wound healing with gene therapy techniques.

Wound healing was variable between participants. We speculate that this may have been due to differences in anatomic location of treated wounds (e.g., back vs. other more easily protected sites), chronicity of wounds, and baseline wound size (greater or less than $100 \mathrm{~cm}^{2}$ ). Participants were allowed to choose which of their eligible wounds were treated and almost all chose their most bothersome areas. For example, participant 6 chose to have his back treated; epidermal sheets on the back are harder to protect from pressure and shear friction, which may have contributed to the observed decreased wound healing at months 6 and 12 (Figure 2). However, despite a poorer wound healing outcome, biopsies of remaining areas of participant 6's back grafts at months 6 and 12 showed very clear molecular correction including $\mathrm{NC} 2 \mathrm{C} 7$ expression and $\mathrm{AF}$ formation. This finding highlights the importance of postoperative care in promoting graft take, even with successful molecular correction.

It appears that very large, older wounds may be more challenging to heal with this technique. At 2 years, only $56 \%$ (10 of 18) of wounds that had been present for 16 years or longer achieved $50 \%$ or greater healing compared with $85 \%$ (17 of 20) of chronic open wounds that were present for less than 15 years. Furthermore, larger baseline wounds (such as those seen in participants 6 and 7) of approximately $105-\mathrm{cm}^{2}$ and $400-\mathrm{cm}^{2}$ wound beds, respectively, required that epidermal sheets be placed side by side in a quilt-like manner onto the prepared wound beds. Therefore, while limited by the small sample size, these data suggest that older wounds and larger wounds may be more challenging to heal.

In a separate but similar study, gene-corrected ( $L A M B 3$ ) epithelial sheets healed wounds for 3.5 years in 1 junctional EB patient. The longer reported durability may have been due to improved basement membrane binding of keratinocytes corrected with laminin 332. Increased durability may also have been due to a higher keratinocyte stem cell population (holoclones) derived from biopsies taken from the patient's palms (17), which was not done in this phase $1 / 2 \mathrm{a}$ trial, given the significant scarring and pseudosyndactyly present in RDEB patients (18). 

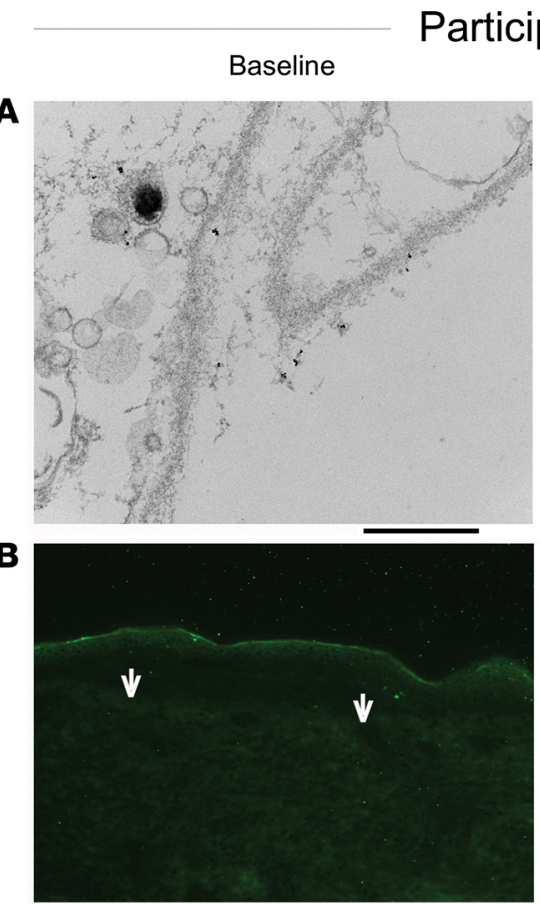

\section{Participant 4}

Baseline

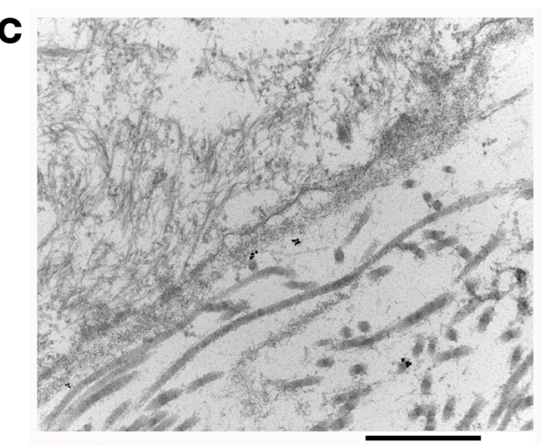

D

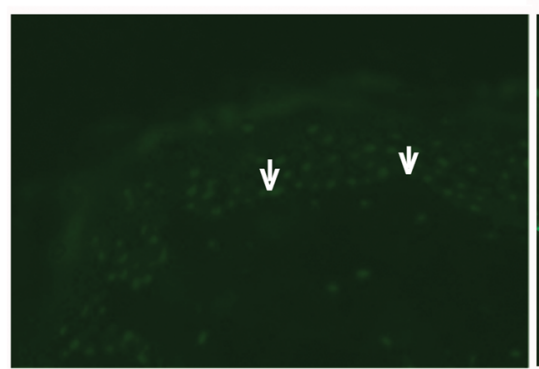

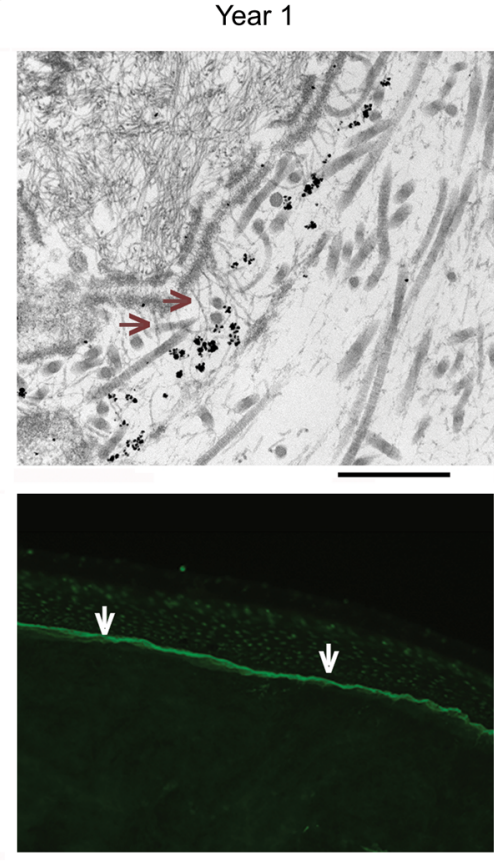

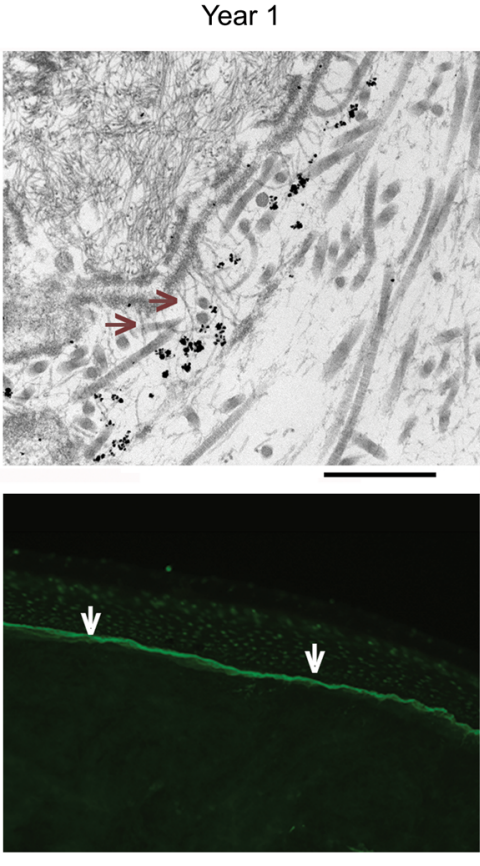

Year 1
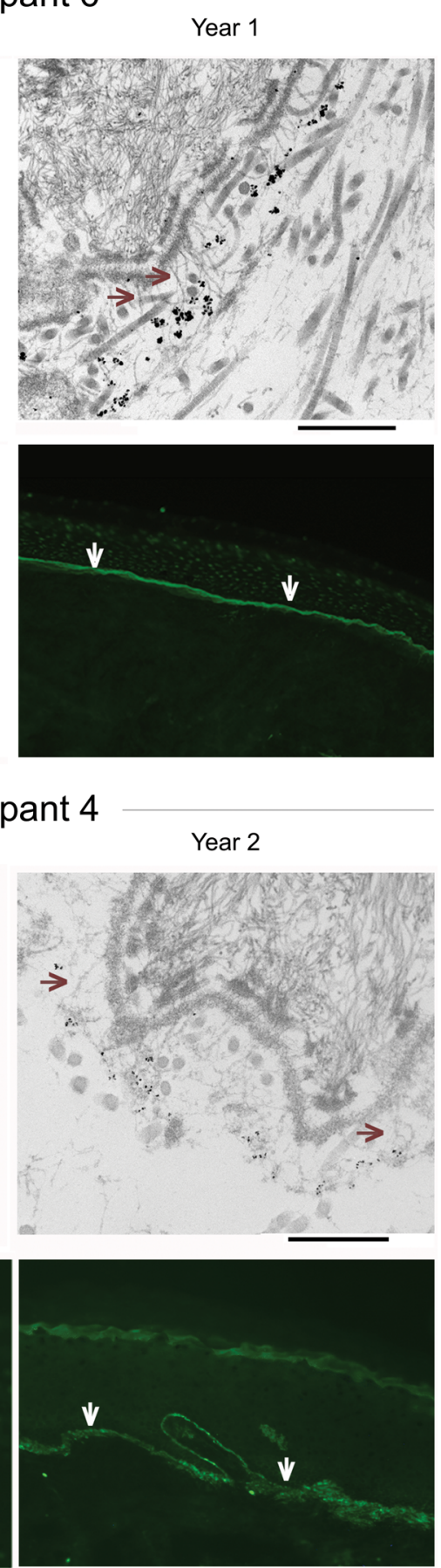

Figure 4. Assessment of molecular correction of $\mathrm{C7}$ in serial biopsies of treated sites (participants 4 and 6 ). Skin biopsies obtained at indicated time points demonstrate presence of $\mathrm{C} 7$ at the dermal-epidermal basement membrane zone. (A and B) Immuno-electron microscopy (IEM) and immunofluorescence (IF), respectively, for participant 6. (C and D) IEM and IF, respectively, for participant 4. (A and C) IEM tissue sections from skin biopsies were labeled en bloc with anti-C7 LH24 monoclonal antibody, followed by anti-mouse IgM-conjugated immunogold particles (black dots, arrows), which decorate anchoring fibrils (AFs). Baseline demonstrates few AFs present for participant 4 . Note that the specimen was split at baseline for participant 6 and thus we cannot comment on presence or absence of AFs. Participant 6 at year 1 and participant 4 at year 2 demonstrate an increased presence of AFs. Scale bars: $500 \mathrm{~nm}$. (B and D) IF from skin biopsies. White arrows indicate the location of the dermal-epidermal junction. Anti-C7 LH24 monoclonal antibody was used to detect the NC2 domain of C7 (green). Linear green staining at the dermal-epidermal junction indicates the presence of $C 7$, which is absent at baseline but observed at 2 years.

Topical tacrolimus was added to minimize potential development of antibodies against C7. We do not believe that this had a role in the observed improvement in wound healing. There are no reports of topical tacrolimus improving wound healing in RDEB. In fact, in clinical studies, tacrolimus has been shown to impair wound healing in normal skin (19). Therefore, improved wound healing may have been more robust had we not used tacrolimus. However, it was determined that the risk of potential impaired healing due to tacrolimus was outweighed by the benefit of minimizing development of autoantibodies, which would likely have also resulted in impairment of wound healing.

In terms of safety, gene therapy risks exposing participants to an unwanted immune reaction against the therapeutic transgene (18) and potential development of cancer through insertional mutagenesis (20). A systemic immune reaction to the gene-correction product (the NC2 domain of C7) would likely manifest as 
worsening blistering at treated sites. If epitope spreading occurred and antibodies were developed against the $\mathrm{NC1}$ domain as well, then diffuse blistering might occur. Fortunately, diffuse new-onset blistering was not observed in any participant.

Serum from all 7 participants was analyzed for tissue-bound antibodies against C7 and treated sites were also biopsied (as permitted by the participants). The minimum number of biopsies was obtained to reduce the potential of harm. While some participants developed trace antibody staining, only 2 participants (participants 2 and 4) developed any IgG beyond trace staining. Participant 4 was noted to have transient circulating antibodies against C7, as described previously (6), and a persistent localized immune response with positive anti-C7 antibodies was noted on DIF of skin biopsies as late as 2 years after treatment. Nevertheless, he did not have systemic worsening of blistering that would warrant concern for a systemic immune response and did not require additional treatment. From the preliminary epitope analysis reported in the previous study (6), participant 4's antibodies were not directed against the NC1 domain (unlike most epidermolysis bullosa acquisita [EBA] patients) but instead against epitopes at or near the NC2 domain that, while present on therapeutic C7, were not likely to be present on the patient's own endogenously produced C7. In this instance, it would not be considered an autoimmune response involving the entire body but instead an alloimmune response that only affected therapeutic C7 confined to portions of the treated sites. This may be why participant 4 did not develop EBA symptoms or increased blistering outside of the treated areas. Participant 2 also developed tissue-bound antibodies. However, these were transient and circulating antibodies were not present, so further epitope analysis was not able to be performed. Neither participant required additional treatment.

The absence of RCR in the serum at all measured time points to date for all participants, with the longest follow-up of 5 years, is reassuring. While 2 participants developed SCCs during the study period, none of the SCCs occurred at treated sites. The available tumor samples were negative for RCR, and both participants were over the age of 30 at the time they developed SCC, which is within the timeframe of heightened concern for SCC development in RDEB patients (2).

Gene-corrected autologous cell therapy (now referred to as EB-101, licensed by Abeona Inc.) demonstrated a favorable safety profile and was shown to have increased long-term healing compared with chronic RDEB wounds, which was associated with long-term C7 molecular expression. EB-101 was granted both Breakthrough Therapy and Regenerative Medicine Advanced Therapy designations by the FDA. This phase $1 / 2$ a study is now closed to enrollment and a phase 3 trial is anticipated to start in late 2019.

\section{Methods}

Study design and participants. Four participants were enrolled in the previously described phase 1 study (6). In 2016, the FDA and the Stanford IRB approved expansion to a phase $2 \mathrm{a}$ study, which enrolled an additional 3 adult participants. All 7 participants were followed for at least 2 and up to 5 years after treatment to date. We measured long-term safety and efficacy data as well as patient-reported outcomes at 3, 6, 9, and 12 months after treatment, and then annually for up to 5 years. Participants continued to receive standardof-care therapies (e.g., iron supplementation, esophageal dilations) during the study.

Participants were age 18 years or older with genetic testing (GeneDx) confirming 2 COL7A1 mutations. Participants had to demonstrate C7 NC1 skin expression $\left(\mathrm{NC1}^{+}\right.$, as determined by Western blot and indirect immunofluorescence [IIF] microscopy), to minimize reaction to the potentially antigenic NC1 domain $(7,21)$. Patients with preexisting circulating or tissue-bound antibodies against C7 on IIF were excluded. Of note, after participant 4 demonstrated anti-C7 antibodies on biopsy of a treated site, a more sensitive analysis was developed using Western blot on pretreatment serum, which demonstrated anti-C7 antibodies at baseline despite negative initial IIF studies (6). Chronic open wounds, defined as wounds present and unhealed for at least 12 weeks, with a total area of at least $100 \mathrm{~cm}^{2}$ were required for enrollment. Of 57 patients screened, 10 gave consent from 2013 to 2017. One patient died due to metastatic SCC prior to treatment, 1 patient did not meet the enrollment criteria for the study, and 1 developed a fever on the day prior to treatment, resulting in cancelation of the procedure (see Supplemental Figure 1).

Study treatment. As previously described (6), autologous keratinocytes were cultured from biopsies from nonwounded, nonscarred skin from each RDEB participant. Keratinocytes were transduced with a retrovirus containing full-length $C O L 7 A 1$ and cultured for approximately 25 days to form epidermal sheets. Surgical placement of 6 epidermal sheets per participant (each $5 \times 7 \mathrm{~cm}$ ) was performed under general anesthesia. During the phase 1 study (participants 1-4), 1 surgically induced wound was created and then treated (site Z) (6). During the phase 2a study (participants 5-7), only noninduced chronic open 
wounds were treated. An untreated chronic open wound was identified to serve as a control wound. Topical tacrolimus was applied to treated sites for participants 5-7 for up to 1 year in an attempt to minimize potential development of antibodies against exogenous C7 (22). All participants were hospitalized postoperatively for up to 8 days to assist with immobilization of the treated sites (Table 1).

Assessments. For the phase 2 a study, the primary endpoint was changed to wound healing as assessed by both IGA and with the Canfield Vectra 3D photography system (Canfield Scientific). IGA was assessed by 2 dermatologists for all wound sites, and wounds were scored as $100 \%$ to $75 \%$ healed, $74 \%$ to $50 \%$ healed, or $49 \%$ to $0 \%$ healed. We compared both methods of evaluating wound healing on the first 36 wounds that were followed for 6 months and found a high degree of correlation between Canfield and IGA (inter-class coefficient $=0.92, P<0.0001)(23)$. Participants enrolled in the phase 1 trial were evaluated by IGA only. Control wounds in the phase 2 a study were evaluated at each visit by IGA and Canfield.

Secondary endpoints included molecular correction as assessed by C7 expression by IIF and presence of C7 localizing to AFs by IEM, at 3, 6, and 12 months after treatment. Additionally, patient-reported outcomes were assessed at each treated site at each study visit. Patients were asked about the presence of pain and itching during the study visit (pain: yes or no, itch: yes or no), durability of grafted areas compared to skin before grafting (more durable, no change, or less durable), and ease of blistering with trauma compared to skin before grafting (more difficult to blister, no change, or blisters easier) at each of the treated sites.

Safety was evaluated by assessing for systemic and/or local immune response, infection, development of SCC, and presence of RCR, as described previously (6). An assay for cytotoxic T cells was performed for only the first 5 participants. Localized immune response was assessed by investigator evaluation of blistering at treated sites and by representative biopsies of selected treated sites. IF and Western blot studies for circulating and immunoreactants bound to the tissue basement membrane zone (IgA, IgG, IgM, and C3) were performed to assess for systemic immune reactions. Testing was performed to evaluate both for an autoimmune reaction (i.e., antibodies against the NC1 domain) as well as an alloimmune reaction (i.e., antibodies against the gene-corrected NC2 domain). Biopsies were obtained at each study visit until 1 year after treatment, after which biopsies were optional. Adverse events were characterized using the National Cancer Institute's Common Terminology Criteria for Adverse Events version 4.0 (https://ctep.cancer.gov/protocolDevelopment/electronic_applications/ctc.htm).

Statistical analysis. Results presented are calculated from the number of treated sites assessed at each designated time point. Fisher's exact test was utilized and all $P$ values are 2-tailed. A $P$ value of less than 0.05 was considered significant. Statistical analysis was performed using SAS 9.4 (SAS Institute Inc.).

Study approval. Study approvals were obtained from the FDA and Stanford IRB prior to participant enrollment as described above. Written informed consent, including consent for photography, was received from participants prior to inclusion in the study. The study was conducted in accordance with the principles of the Declaration of Helsinki.

\section{Author contributions}

SE, MB, JYT, and MPM wrote the manuscript. MB, CT, JN, ESG, JYT, and MPM conducted the study and acquired data. LT, PK, RKK, LKF, HPL, MPM, and ASC provided direct patient care. SE, MB, CT, JYT, and MPM analyzed data. AP, NTN, ZS, DRK, KR, and MPM performed diagnostic studies and analysis.

\section{Acknowledgments}

We gratefully acknowledge the study participants as well as study staff, physicians, nurses, and other personnel at Stanford University and Stanford Children's Hospital. Research funding is gratefully acknowledged from the Epidermolysis Bullosa Medical Research Foundation (grant to support EB research), the Epidermolysis Bullosa Research Partnership, and NIH grant R01 AR055914 (initial grant to support phase 1 trial). Support was also provided by the Office of Research and Development and the Palo Alto Veteran's Affairs Medical Center (salary support for MPM) and the Dermatology Foundation (salary support for AC).

Address correspondence to: Jean Y. Tang, 450 Broadway Street, Pavilion C, MC: 5334, Redwood City, California 94063, USA. Phone: 650.721.7190; Email: tangy@stanford.edu. Or to: M. Peter Marinkovich, 455 Broadway Street, Discovery Hall, Room D126, MC: 8843, Redwood City, California 94063, USA. Phone: 650.498.5425; Email: mpm@stanford.edu. 
1. Shinkuma S. Dystrophic epidermolysis bullosa: a review. Clin Cosmet Investig Dermatol. 2015;8:275-284.

2. Marinkovich M. Chapter 62. Inherited epidermolysis bullosa. In: Goldsmith LA, Katz SI, Gilchrest BA, Paller AS, Leffell DJ, Wolff K, eds. Fitzpatrick's Dermatology in General Medicine. 8th ed. New York, New York, USA: McGraw-Hill; 2012.

3. Fine JD, et al. Inherited epidermolysis bullosa: updated recommendations on diagnosis and classification. J Am Acad Dermatol. 2014;70(6):1103-1126.

4. Hashmi S, Marinkovich MP. Molecular organization of the basement membrane zone. Clin Dermatol. 2011;29(4):398-411.

5. Sebastiano V, et al. Human COL7A1-corrected induced pluripotent stem cells for the treatment of recessive dystrophic epidermolysis bullosa. Sci Transl Med. 2014;6(264):264ra163.

6. Siprashvili Z, et al. Safety and wound outcomes following genetically corrected autologous epidermal grafts in patients with recessive dystrophic epidermolysis bullosa. JAMA. 2016;316(17):1808-1817.

7. Gorell ES, Nguyen N, Siprashvili Z, Marinkovich MP, Lane AT. Characterization of patients with dystrophic epidermolysis bullosa for collagen VII therapy. Br J Dermatol. 2015;173(3):821-823.

8. James WD, Berger TG, Elston DM, Neuhaus IM. Andrews' Diseases of the Skin. 12th ed. Amsterdam, Netherlands: Elsevier; 2015.

9. Danial C, et al. Prevalence and characterization of pruritus in epidermolysis bullosa. Pediatr Dermatol. 2015;32(1):53-59.

10. Solis D, et al. Defining chronic wound types in recessive dystrophic epidermolysis bullosa patients for clinical outcome assessment. J Invest Dermatol. 2018;138(5):S97.

11. Falabella AF, Valencia IC, Eaglstein WH, Schachner LA. Tissue-engineered skin (Apligraf) in the healing of patients with epidermolysis bullosa wounds. Arch Dermatol. 2000;136(10):1225-1230.

12. Fivenson DP, Scherschun L, Choucair M, Kukuruga D, Young J, Shwayder T. Graftskin therapy in epidermolysis bullosa. $J A m$ Acad Dermatol. 2003;48(6):886-892.

13. McGrath JA, et al. Cultured keratinocyte allografts and wound healing in severe recessive dystrophic epidermolysis bullosa. J Am Acad Dermatol. 1993;29(3):407-419.

14. Siprashvili Z, Nguyen NT, Bezchinsky MY, Marinkovich MP, Lane AT, Khavari PA. Long-term type VII collagen restoration to human epidermolysis bullosa skin tissue. Hum Gene Ther. 2010;21(10):1299-1310.

15. Marinkovich MP, Tang JY. Gene therapy for epidermolysis bullosa. J Invest Dermatol. 2019;139(6):1221-1226.

16. Lwin SM, et al. Safety and early efficacy outcomes for lentiviral fibroblast gene therapy in recessive dystrophic epidermolysis bullosa. JCI Insight. 2019;4(11):126243.

17. Mavilio F, et al. Correction of junctional epidermolysis bullosa by transplantation of genetically modified epidermal stem cells. Nat Med. 2006;12(12):1397-1402.

18. De Luca M, Pellegrini G, Mavilio F. Gene therapy of inherited skin adhesion disorders: a critical overview. Br J Dermatol. 2009;161(1):19-24.

19. Schäffer MR, Fuchs N, Proksch B, Bongartz M, Beiter T, Becker HD. Tacrolimus impairs wound healing: a possible role of decreased nitric oxide synthesis. Transplantation. 1998;65(6):813-818.

20. Howe SJ, et al. Insertional mutagenesis combined with acquired somatic mutations causes leukemogenesis following gene therapy of SCID-X1 patients. J Clin Invest. 2008;118(9):3143-3150.

21. Lapiere JC, et al. Epitope mapping of type VII collagen. Identification of discrete peptide sequences recognized by sera from patients with acquired epidermolysis bullosa. J Clin Invest. 1993;92(4):1831-1839.

22. Wallin EF, Hill DL, Linterman MA, Wood KJ. The calcineurin inhibitor tacrolimus specifically suppresses human T follicular helper cells. Front Immunol. 2018;9:1184.

23. Dutt-Singkh Y, et al. 50\% wound healing correlates with RDEB patient reported outcomes in pain, itch and skin durability. J Invest Dermatol. 2018;138(5):S56. 